Andreas Rohrwasser - Shuhua Zhang • Harrison F. Dillon

Ituro Inoue • Christopher W. Callaway • Elaine Hillas

Jean-Marc Lalouel

\title{
Contribution of Sp1 to initiation of transcription of angiotensinogen
}

Received: January 16, 2002 / Accepted: February 25, 2002

\begin{abstract}
Several genetic polymorphisms have been identified in the proximal promoter of angiotensinogen $(A G T)$. Gene titration experiments in transgenic animals have demonstrated that small increases in the basal expression of AGT can lead to elevated blood pressure. The direct proof that promoter variants of AGT can lead to elevated blood pressure will ultimately require the development of specific animal models. Before such work can be contemplated, however, a formal understanding of the mechanisms controlling transcriptional activation of AGT needs to be developed. Analysis of DNA-protein interactions in vitro and transactivation experiments in cultured cells reveal the critical role of an $\mathrm{Sp} 1$ binding site immediately upstream of the TATA box of $A G T$ in both mouse and human. Both sites are required for transcription initiation in the mouse. By contrast, a minimal human $A G T$ promoter can initiate transcription in the absence of either this Sp1 site or the TATA box, albeit at a lower level. Further analysis and consideration of these interspecific differences will be essential for the development of meaningful animal models to probe the mechanism by which AGT may predispose to human essential hypertension.
\end{abstract}

Key words Angiotensinogen $\cdot$ Transcription $\cdot \mathrm{Sp} 1 \cdot$ Minimal promoter $\cdot$ Transcription factors $\cdot$ DNA binding $\cdot$ Hypertension

A. Rohrwasser $\cdot$ S. Zhang $\cdot$ H.F. Dillon - I. Inoue $\cdot$ C.W. Callaway $\cdot$

E. Hillas - J.-M. Lalouel

Department of Human Genetics, University of Utah School of Medicine, Salt Lake City, UT, USA

S. Zhang $\cdot$ C.W. Callaway $\cdot$ E. Hillas $\cdot$ J.-M. Lalouel

Howard Hughes Medical Institute, University of Utah School of Medicine, Salt Lake City, UT, USA

J.-M. Lalouel ( $\square)$

Eccles Institute of Human Genetics, University of Utah Health

Sciences Center, Salt Lake City, UT 84112, USA

Tel. +1-801-581-4311; Fax +1-801-587-9045

e-mail: jml@howard.genetics.utah.edu

\section{Introduction}

Little is known about the genetic determinants of essential hypertension in humans, despite extensive investigations in animal models of high blood pressure (Fukamizu and Murakami 1997) and significant advances in the genetics of rare mendelian forms of hypertension (Lifton et al. 2001). The combination of complex causation and etiological heterogeneity generates a unique challenge in the genetics of common human disorders. Genetic linkage and association studies in two major ethnic groups suggest a role for angiotensinogen $(A G T)$ in human essential hypertension (Hata et al. 1994; Jeunemaitre et al. 1992). AGT is the hormone precursor of angiotensin II, which affects both vascular structure and sodium homeostasis.

The association observed between both plasma AGT and hypertension and a molecular variant encoding a threonine instead of a methionine at residue 235, denoted T235, could either be causal or could serve as a marker for an unknown causal factor in the gene. Recent data reveal that, with few exceptions, genes carrying allele T235 exhibit an adenine six residues upstream of the initiation site of transcription, denoted A(-6), and M235 alleles have a guanine at this site, or G(-6) (Inoue et al. 1997). Binding studies and transactivation experiments in cultured human cells revealed that this polymorphism in the proximal promoter of AGT affects specific interactions with nuclear proteins and leads to significant differences in promoter activity in vitro (Inoue et al. 1997). By establishing a direct causal link between AGT copy number and both plasma AGT and blood pressure, gene titration experiments in transgenic mice have provided direct proof that a modest but sustained increase in AGT expression can lead to chronic elevation of arterial pressure (Kim et al. 1995).

Given these observations, our working hypothesis is that molecular variation in the proximal promoter of $A G T$ can impart differences in the regulatory response of the gene to changes in dietary sodium. The actual molecular mechanism, the relevant tissue site, and the physiological relationship of this genetic variation to blood pressure regulation 
remain to be characterized. The ultimate proof of a causal link between genetic variation and physiological change will have to rest on gene manipulation in experimental animals.

An initial step in this direction consists of identifying critical elements controlling AGT transcription in both human and mouse. Previous work with the human promoter has yielded intriguing results. A minimum promoter spanning nucleotides -32 to +44 and including the TATA box appeared as active as the -1200 to +44 segment (Fukamizu et al. 1990). In contrast, a promoter segment spanning nucleotides -32 to +22 appeared inactive (Tamura et al. 1994). Specific elements were identified on either side of the TATA box, initially without clue as to the actual transacting factors involved (Tamura et al. 1993, 1994). Alignment of the human and mouse core promoters reveal conserved as well as nonconserved sequence segments, indicating the need for a formal comparative study of these two promoters. The following work documents the absolute requirement for functional interactions between $\mathrm{Sp} 1$ and TATA-binding protein in the initiation of transcription of AGT in the mouse, whereas additional alternate initiation pathways may exist in humans.

\section{Methods}

Cell culture

Mouse and human hepatoma cells (Hepa1-6 and HepG2) were maintained in DMEM containing 10\% fetal bovine serum, $5 \mathrm{mM}$ hydroxyethylpiperazine ethanesulfonic acid (HEPES), $100 \mathrm{IU} / \mathrm{ml}$ penicillin and $100 \mu \mathrm{g} / \mathrm{ml}$ streptomycin. The cells were kept in $5 \% \mathrm{CO}_{2} / 95 \%$ air at $37^{\circ} \mathrm{C}$. The conditionally immortalized murine cell line of proximal tubule epithelium, $t s \mathrm{MPT}$, was maintained as described previously (Loghman-Adham et al. 1997).

Preparation of nuclear extracts

Nuclear extracts from Hepa1-6 cells, tsMPT cells, and HepG2 cells were prepared using modifications of the procedure of Dignam (Dignam et al. 1983). Final protein concentrations were between 2 and $5 \mathrm{mg} / \mathrm{ml}$.

Electrophoretic mobility shift assay (EMSA)

Synthetic double-stranded AGT promoter sequences (Table 1) were phosphorylated at both ends using T4 polynucleotide kinase and $\left(\gamma_{-}{ }^{32} \mathrm{P}\right)$ ATP (NEN-Life Science,

Table 1. Mouse and human oligonucleotides

Mouse oligonucleotides for electrophoretic gel mobility shift assays

\begin{tabular}{lll}
\hline Oligonucleotide & Position $5^{\prime}$ & Sequence $5^{\prime}$ to $3^{\prime}$ \\
\hline 6133 & -61 & CCCAGGAATAGATCCATCTCCACCCCTTGA \\
6133mutGATA & -61 & CCCAGGATTTTATCCATCTCCACCCCTTGA \\
6133mutSp1 & -61 & CCCAGGAATAGATCCATCT $A A G A A C C T T G A$ \\
\hline
\end{tabular}

Sense-oligos are displayed; mutagenic sequences are indicated in italics

Mouse oligonucleotides for mutagenic constructs

\begin{tabular}{lll}
\hline Oligonucleotide & Position $5^{\prime}$ & Sequence 5' to $3^{\prime}$ \\
\hline 61wt & -61 & ggtggtaccCCAGGAATAGATCCATCTCC \\
61mutTATA & -61 & $\begin{array}{l}\text { ggtggtaccCCAGGAATAGATCCATCTCCACCCCTT } \\
\text { GAGGCATCTTAAGCCTGCTTGGCTCACCAGGGGATAGC }\end{array}$ \\
61mutSp1/TATA & -61 & $\begin{array}{l}\text { ggtggtaccCCAGCAATAGATCCATCTTTGTTTCTTG } \\
\text { AGGCATCTTAAGCCTGCTTGGCTCACCAGGGGATAGC } \\
\text { 61mutSp1 }\end{array}$ \\
\hline
\end{tabular}

Italics, Mutagenic sequence; small caps bases, nonpromoter sequence with $K p n I$ restriction site underlined

Human oligonucleotides for mutagenic constructs

\begin{tabular}{lll}
\hline Oligonucleotide & Position $5^{\prime}$ & Sequence $5^{\prime}$ to $3^{\prime}$ \\
\hline 34wt & -34 & ataggtaccGCTATAAATAGGGCCTCGTGA \\
70wt & -70 & ggtggtaccCAGGCAGCCTGGGAACAGCTCC \\
70mutTATA & -70 & ataggtaccCAGGCAGCCTGGGAACAGCTCCATC \\
CCCACCCCTCAGCGCATCTTAGGGCCTCGTGACCCGGCC & ataggtaccCAGGCAGCCTGGGAACAGCTCCATC $A$ \\
70mutSp1/TATA & -70 & $\begin{array}{l}\text { AAACCCCTCAGCGCATCTTAGGGCCTCTGACCCGGC } \\
\text { ataggtaccCAGGCAGCCTGGGAACAGCTCCATC } A\end{array}$ \\
70mutSp1 & -70 & $\begin{array}{l}\text { AAACCCCTCAGCTATAAATAGGGCCTCGTGA } \\
\text { cctataagcttccggCTTACCTTCTGCTGTAGTA }\end{array}$ \\
\hline
\end{tabular}

Small caps bases, underlined, Nonpromoter sequence with KpnI restriction site; underlined, mutagenic sequence 
Boston, MA, USA). Nuclear extracts or purified proteins (Promega, Madison, WI, USA) were preincubated for $15 \mathrm{~min}$ on ice in a $20-\mu \mathrm{l}$ reaction mixture containing $1.5 \mu \mathrm{g}$ poly(dI-dC) (Amersham Pharmacia Biotech, New Jersey, NY, USA), $10 \%$ glycerol, $20 \mathrm{mM}$ HEPES (pH 7.9), $50 \mathrm{mM}$ $\mathrm{KCl}, 0.5 \mathrm{mM}$ dithiothreitol (DTT), $0.2 \mathrm{mM}$ ethylenediaminetetra acetate (EDTA), and $6.25 \mathrm{mM} \mathrm{MgCl}_{2}$ in the presence or absence of 50- or 100-fold molar excess of specific or nonspecific double-stranded competitor DNA or specific or control antisera. Reactions with purified Sp1 or Sp3 contained $0.01 \%$ Tween-20 to prevent protein aggregation. A 200 -fmol aliquot of ${ }^{32} \mathrm{P}$-labeled double-stranded oligonucleotide probe was added, and the reaction was continued on ice for $15 \mathrm{~min}$. The reactions were loaded onto $5.5 \%$ nondenaturing polyacrylamide gels in $0.5 \times \mathrm{TBE}(90 \mathrm{mM}$ Tris- $\mathrm{HCl}$ at $\mathrm{pH} 8.0,89 \mathrm{mM}$ boric acid, $2 \mathrm{mM}$ EDTA) and electrophoresed at $200 \mathrm{~V}$ for $2 \mathrm{~h}$ at room temperature. Antibodies against Sp1, Sp3, GATA-1, GATA-2, NF-1, AP-1, AP-2, and control-IgG, as well as purified oligonucleotides containing the consensus binding sites for Sp1, AP-2, NF-1, Oct-1, C/EBP, CRE, were obtained from Santa Cruz Biotechnology Inc, Santa Cruz, CA, USA.

\section{Plasmid construction}

Mouse AGT promoter fragments were generated by polymerase chain reaction (PCR) using a genomic clone as template, primers with overhanging restriction sites $\left(5^{\prime} \mathrm{KpnI}\right.$ and $3^{\prime}$ HindIII; Table 1), and Pfu-polymerase to minimize misincorporation. PCR fragments were digested with $K p n \mathrm{I}$ and HindIII and subcloned into KpnI- and HindIIIdigested pGL3-Basic, also double digested with KpnI and HindIII. The integrity of the promoter sequences was verified by DNA sequencing. All plasmids used for transfection experiments were isolated from the DM-1 Escherchia coli strain $\left(\mathrm{dam}^{-} / \mathrm{dcm}^{-}\right)$to exclude the possibility of differential methylation effects.

AGT promoter construct $2(-61$ to +44$)$ was used as a template to generate Sp1, TATA, and Sp1/TATA mutant constructs by oligonucleotide-directed PCR mutagenesis using sense primers with the mutations. Once the mutant AGT promoter fragments were obtained by PCR, they were double digested with KpnI and HindIII and ligated into double-digested pGL3-Basic.

Human AGT (hAGT) promoter fragments were also generated by PCR using a previously generated hAGT promoter construct as template, primers with overhanging restriction sites (5' KpnI and 3' HindIII; Table 1), and Pfupolymerase to minimize misincorporation. Mutant hAGT promoter constructs were created by oligonucleotidedirected PCR mutagenesis. Double-digested PCR products were cloned into KpnI- and HindIII-digested pGL3-Basic, as described for the murine AGT promoter constructs.

\section{DNA transfection and reporter gene assays}

Transfection experiments were performed using the Promega Tfx-system (Promega) and the pGL3-Basic re- porter system (Promega) lacking eukaryotic promoter and enhancer sequences. PCR-generated AGT promoter sequences were cloned into pGL3-Basic using KpnI and HindIII sites of the multiple cloning site $5^{\prime}$ of the fireflyluciferase reporter gene. pGL3-Control (Promega) with the SV40 promoter and enhancer sequence $5^{\prime}$ of the fireflyluciferase reporter gene served as a positive control, and pGL3-Basic without any eukaryotic promoter and enhancer sequence served as a negative control. The pRL-SV40 plasmid (Promega) was cotransfected in all experiments. Coexpression of Renilla luciferase under the control of an SV40 promoter and enhancer was used to standardize transfection efficiency. Cells (Hepa1-6 or HepG2) were seeded in 12-well plates $\left(6 \times 10^{5}\right.$ per well) coated with collagen type I $48 \mathrm{~h}$ prior to transfection. Optimal transfection results were achieved with $1.45 \mathrm{pmol}$ of AGT promoter plasmid DNA per well, the Tfx-10 cationic lipid mixture at a $3: 1$ DNA:lipid ratio, and an incubation time of $2 \mathrm{~h}$. pRL-SV40 ( 0.145 pmol per well) was used to standardize for transfection efficiency. At $24 \mathrm{~h}$ post-transfection, the cells were washed with phosphate-buffered saline and lysed in $100 \mu \mathrm{l}$ passive lysis buffer (Promega). Firefly and Renilla luciferase reporter gene activities were measured using the Dual Luciferase Activity Assay kit (Promega) and a Turner TD20e luminometer (Promega). All transfections were performed in triplicate and repeated in three independent experiments with two independent plasmid DNA preparations. All transfection results are expressed as the ratio of firefly luciferase activity over Renilla luciferase activity. HepG2 cells were transfected using the cationic lipid mixture Tfx-20 (Promega).

DNA sequence analysis

The 5' promoter sequence of the mouse AGT gene (129/ $\mathrm{SvEv}$ ) obtained from a genomic library screen cloned into pBluescript was used for sequencing. Sequencing was performed using ABI 377 automated sequencing and dyeterminator technology. Sequencing information was generated by "walking," using primers derived from the sequenced $5^{\prime}$ promoter region. Sequence information was verified by sequencing sense and antisense strands.

Identification of putative promoter elements

Putative transcription factor response elements were identified using TransFact and TF algorithms (Heinemeyer et al. 1999, 1998).

\section{DNase I footprint analysis}

Fragments of the AGT promoter $(-100$ to +44$)$ were generated by PCR using $\gamma{ }^{32}$ P-labeled forward or reverse primers. After spin dialysis (Centricon-100) and concentration, the probes were incubated with 2 to $15 \mathrm{fpU}$ (footprinting unit, fpU) of recombinant human Sp1 (Promega) in a 50- $\mu$ l reaction volume containing $12 \mathrm{mM}$ HEPES (pH 7.9) $5 \mathrm{mM}$ 
$\mathrm{CaCl}_{2}, 5 \mathrm{mM} \mathrm{MgCl}_{2}$, and 5 to $250 \mathrm{ng}$ DNase I. The reaction was stopped by the addition of $100 \mu \mathrm{l}$ stop solution $(12 \mathrm{mM}$ HEPES at $\mathrm{pH} 7.9,0.6 \mathrm{M}$ sodium acetate at $\mathrm{pH} 7,0.5 \%$ sodiumdodecyl sulfate, $0.1 \mathrm{mM}$ EDTA, and $20 \mu \mathrm{g}$ of tRNA). The DNA was extracted twice with phenol-chloroform $(1: 1, \mathrm{vol} / \mathrm{vol})$ and precipitated in 2.5 volume of $100 \%$ ethanol prior to electrophoresis on a $6 \%$ polyacrylamide $/ 8 \mathrm{M}$ urea sequencing gel. To accurately determine the positions of protected bases, we prepared G, A, C, T sequence reactions (Maxam and Gilbat 1980) and ran them as markers in parallel lanes.

\section{Results}

The -61 to +44 promoter construct is sufficient to achieve promoter activity

To identify the minimal promoter length necessary for AGT transcription, we transfected a series of staggered mouse AGT promoter constructs into the murine kidney proximal tubule cell line $t s$ MPT (Loghman-Adham et al. 1997) and the murine hepatoma cell line Hepa1-6 (Fig. 1). Both cell lines were previously shown to express angiotensinogen.

In both cell lines, a minimal promoter construct ( -32 to +44 ) with only the TATA box showed no promoter activity when compared with the promoterless control reporter gene. Promoter activity was obtained with a minimal promoter spanning -61 to +44 . Further $5^{\prime}$ extension resulted in a reduction of reporter gene activity. In $t s \mathrm{MPT}$ cells, promoter activity dropped to background levels, whereas in Hepa1-6 cells, AGT promoter activity was reduced by more than $70 \%$. In contrast to the region between -32 and -61 , several elements have been identified in the region between -91 and -61, including an AGE2 element (Tamura et al. 1993, 1994), a SOAP (suppression or activation protein) binding site (Brasier et al. 1989), and an Sp1 binding site (Congiu et al. 1992). AGT promoter activity increased again with construct 4 ( -412 to +44 , Fig. 1$)$ to levels close to those observed with construct 2 . In summary, these re- sults confirm previous observations that a truncated AGT $(-412)$ promoter is sufficient to achieve maximal promoter activity (Brasier et al. 1989). These results also demonstrate that a minipromoter ranging from -61 to +44 is sufficient for AGT promoter activity in tsMPT and Hepa1-6 cells in vitro.

To identify putative transcription factor binding sites within the region between -61 and the TATA-box, we used computer-aided sequence homology search algorithms and found several potential candidate response elements: GATA ( -56 to -51$)$, mycCF1 ( -53 to -48$)$, and two NF1 half sites $(-50$ to -47 and -44 to -41$)$. Additionally, a potential intermediate affinity Sp1 site (Jones et al. 1986; Kriwacki et al. 1992) ( -47 to -38$) 5$ bp adjacent to the TATA box was identified.

The promoter sequence immediately $5^{\prime}$ to the TATA box is conserved between human, mouse, and rat AGT and contains an Sp1 binding site

Sequence comparison between human and rodent proximal AGT promoter shows regions with a high degree of sequence homology interspersed with regions of low or no homology (Fig. 2). In particular, a segment directly upstream of the TATA box, encompassing nucleotides -37 to -53 , exhibits conservation between human and rodents. In the human sequence, we observed an intermediate affinity Sp1 binding site between -38 and -47 . The same region of the mouse gene deviates by one ( $-46 \mathrm{C}$-to-T substitution), whereas the rat sequence shows two substitutions ( $-46 \mathrm{C}$ to-T substitution and $-45 \mathrm{C}$-to-T substitution).

In vitro binding experiments demonstrated direct binding between Sp1 and the region between -61 and -32 (Fig. $3 A)$. EMSAs using an oligonucleotide probe spanning the region between -61 and -32 (6132) and Hepa1-6 nuclear extracts show the characteristic Sp1 binding pattern with Sp1 as the top band, Sp3 as the second band, and a nonspecific third band. Lanes 4 to 7 show various competition experiments. Competition with excess unlabeled 6132 resulted in complete loss of binding (lane 4); the same was
Fig. 1. Analysis of the promoter of mouse angiotensinogen $(A G T)$ in $t s$ MPT and Hepa1-6 cells. Results are expressed as ratios of Firefly luciferase activity over SV40-Renilla luciferase activity. Black bars indicate results in tsMPT cells; white bars show results for Hepa1-6 cells. Numbers indicate the construct and the corresponding 5' sequence, respectively

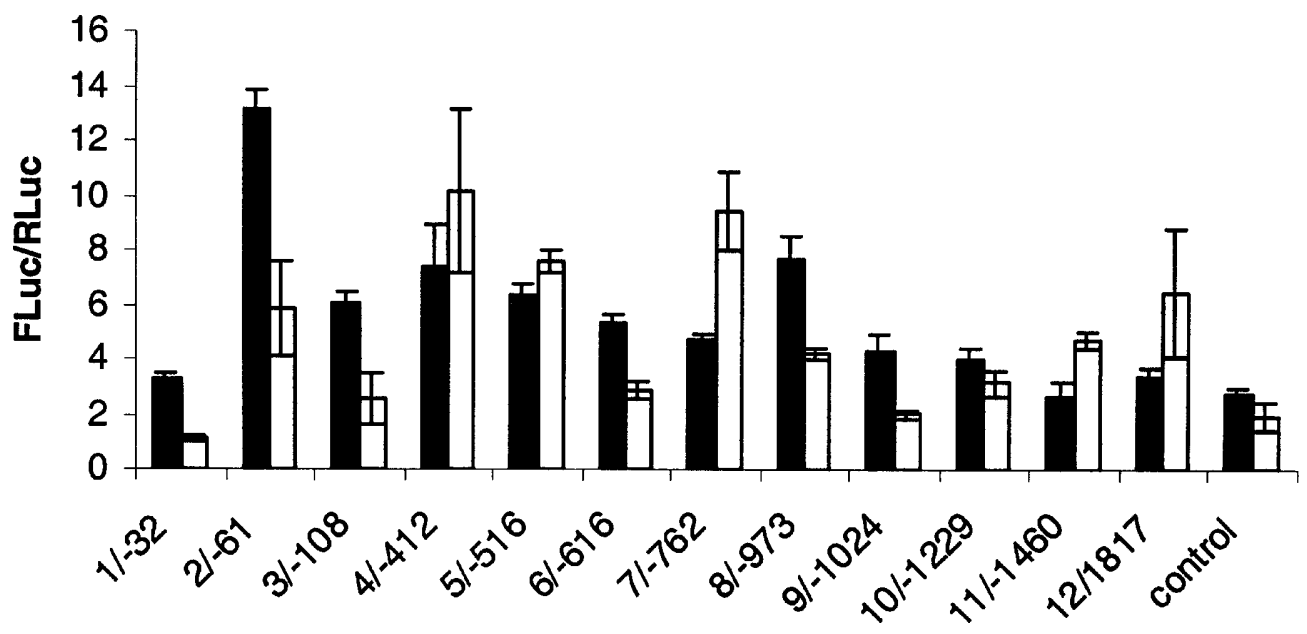




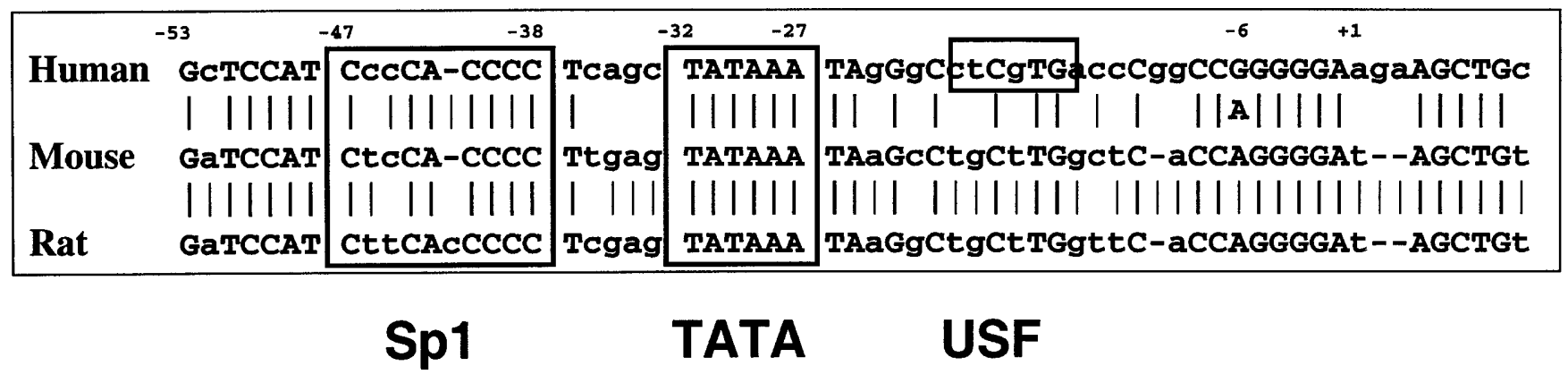

Fig. 2. Comparison of the promoter sequences of human, mouse, and rat AGT. Sequences of the human, mouse, and rat AGT promoters are displayed from -53 to +9 . Consensus binding sites are boxed

observed with excess Sp1 consensus oligonucleotide (lane 5). An oligonucleotide probe, including a mutation in the Sp1 binding domain of 6132, failed to compete in gelshift experiments (lane 6). Competition was also observed with a probe with a mutation in the putative GATA motif (mGATA, lane 7). Preincubation with anti-Sp1 antibodies resulted in the formation of a specific high-molecularweight complex (lane 8), which was not detected when only control IgG was used (lane 1). Identical patterns were observed when $t$ MPT nuclear extracts were used (data not shown).

The same binding patterns were observed when nuclear extracts prepared from the human hepatoma cell line HepG2 were used (Fig. 3B).

Recombinant human Sp1 binds to the mouse Sp1 binding site between -51 and -34

Because the Sp1 element identified is in very close proximity to the TATA box, we investigated the region of Sp1DNA contact more thoroughly by DNase I protection experiments. Protection studies using recombinant human Sp1 showed a protected region from -34 to -51 (Fig. 4), suggesting no overlap with the TATA box.

The TATA box and the adjacent Sp1 site are necessary but not sufficient for murine AGT transcription

To demonstrate the functional significance of the Sp1 binding site immediately $5^{\prime}$ of the TATA box and to determine whether the Sp1 binding site would be sufficient to initiate transcription, we generated a series of mutant AGT promoter constructs, with mutations in 1) the Sp1 binding site, 2) the TATA box, and 3) both the Sp1 binding site and the TATA box. We performed transfection experiments in Hepa1-6 (Fig. 5A) and $t s$ MPT cells (data not shown), comparing reporter gene activities of the wild-type promoters $(-32$ to +44 and -61 to +44$)$ to the promoterless control and the various mutant constructs. In both cell lines, a minimal promoter, including the TATA box, is not active compared with the promoterless control, indicating that the TATA box alone is not sufficient to initiate transcription of murine $A G T$ gene in vitro. A mutation inactivating the TATA box (mTATA) resulted in complete loss of reporter gene activity and demonstrates the functional necessity of the TATA box for AGT transcription. The Sp1 site ( -47 to +38 ) is necessary but also not sufficient to induce AGT transcription. A mutation in the Sp1 element (mSp1) also resulted in the complete loss of reporter gene activity. Mutations in the Sp1 site and the TATA box (mTATAmSp1) also eliminated reporter gene activity. These results demonstrate that 1) a minimal AGT promoter construct including only the TATA box is not active, 2) within the context of a short AGT promoter, both the Sp1 binding site and the TATA box are necessary for AGT transcription, and 3) the $\mathrm{Sp} 1$ binding site is not sufficient to induce transcription of murine AGT in vitro.

A minimal human AGT promoter construct is active and mutations in the TATA box and Sp1 site do not cause complete transcriptional inactivation

To assess whether the proximal promoters of mouse and human AGT require the same transacting factors, we tested a series of human promoter constructs, spanning the region from -70 to +44 , with mutations in 1 ) the $\mathrm{Sp} 1$ binding site, 2) the TATA box, 3) the Sp1 binding site and the TATA box, and 4) a minimal promoter construct spanning the region from -32 to +44 (Fig. $5 \mathrm{~B}$ ). In transfection experiments in the human hepatoma cell line HepG2, the promoter construct, including the Sp1 site, was more active than a minimal construct including only the TATA box (construct 1) and all other constructs tested. Mutational inactivation of the Sp1 site or the TATA box led to significant reduction of reporter gene activity, but did not result in complete inactivation of the promoter. In vitro binding studies and competition experiments demonstrated that all generated mutations abrogated binding in vitro (data not shown). All promoter constructs showed significantly more transcriptional activity when compared with the promoterless control. These results suggested that, in contrast to the mouse AGT promoter, a minimal human AGT promoter $(-32$ to +44$)$ remains functionally active in the absence of either or both of the Sp1 and TATA binding site. 

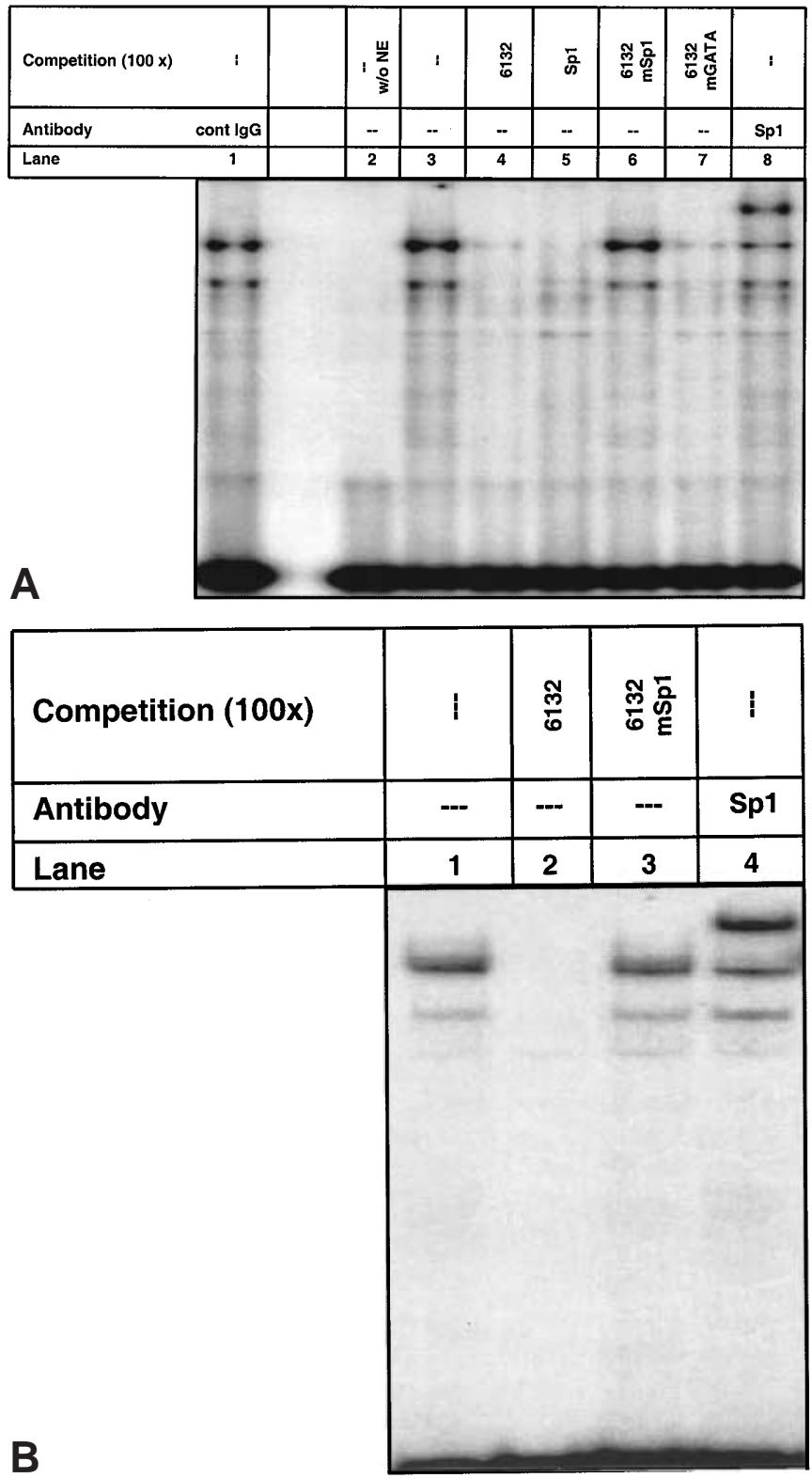

Fig. 3A,B. Sp1 binding to the murine and human AGT promoter in vitro. A Binding of Sp1 and Sp3 to the murine AGT promoter using Hepa1-6 nuclear extracts and an oligonucleotide probe spanning the promoter between -61 and -32 (6132) by electrophoretic gel mobility assay. B Binding of Sp1 to the human AGT promoter using HepG2 nuclear extracts and an oligonucleotide probe spanning the human promoter between -61 and -33 (6132) by electrophoretic gel mobility assay

\section{Discussion}

A common $A G T$ gene polymorphism (M235T) is associated with essential hypertension and preeclampsia (Hata et al. 1994; Jeunemaitre et al. 1992; Ward et al. 1993). This polymorphism was shown to be in very tight linkage disequilibrium with a mutation in the promoter of AGT, A(-6). Recent functional studies showed that the $\mathrm{A}(-6)$ promoter mutation leads to increased $A G T$ T235 transcription in vitro relative to its $\mathrm{G}(-6)$ counterpart (Inoue et al. 1997).

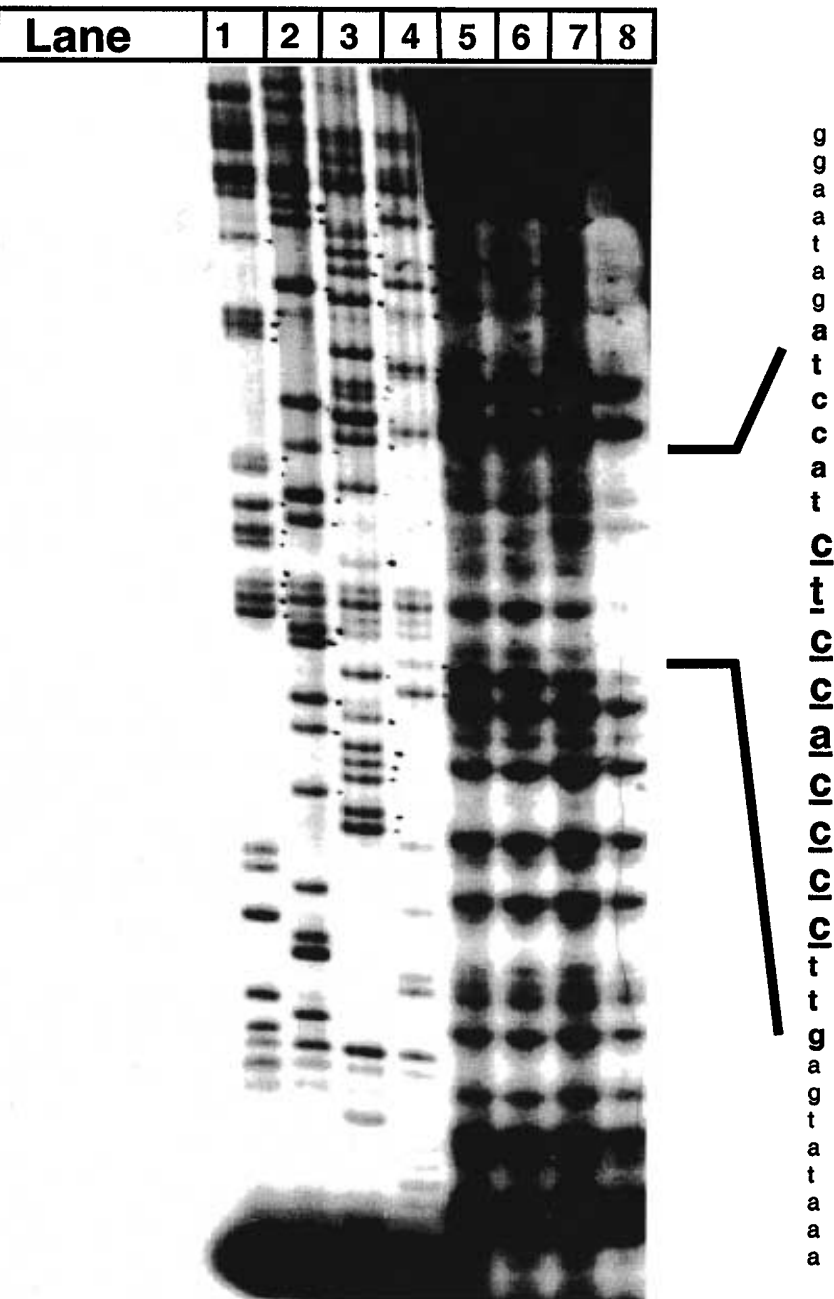

Fig. 4. Mapping of Sp1 binding to the mouse AGT promoter by DNase I protection experiments. Electrophoresis of Maxam-Gilbert sequencing reactions was used to identify protected areas (lanes 1-4). Increasing amounts ( 2 to 15 footprinting unit, fpU) of recombinant human Sp1 result in specific DNA protection (lanes 5-8)

Furthermore, elevated -6(A)/235T-AGT expression was shown in decidual spiral arteries of preeclamptic females (Morgan et al. 1997), providing a direct link between increased tissue 235T expression and hypertensive disorders.

We have analyzed the proximal promoter of murine $A G T$ to identify enhancer and promoter elements necessary for transcriptional activation of the murine $A G T$ gene. Detailed information about basic, stimulated, or tissuespecific transcription of mouse $A G T$ is necessary to establish the basis for future gene replacement experiments. The goal of such gene targeting studies is to assess in vivo effects of the AGT polymorphisms identified in human hypertensive subjects. Such studies require detailed understanding of the functional differences and similarities between the murine and human AGT promoters.

To establish the minimal length of a functional murine AGT promoter, we generated a series of staggered promoter constructs. Our results showed that a minimal murine AGT promoter $(-32$ to +44$)$ with only the TATA box is inactive. Promoter activity is achieved with a minipromoter 
Fig. 5A,B. Analysis of the mouse AGT promoter by sitedirected mutagenesis and transfection analysis. A A minimal promoter $(-32$ to +44$)$ is inactive compared with a promoterless control (promoterless cont). $5^{\prime}$ extension to $-61(-61$ to +44 ), including the Sp1 site, results in promoter activation $(\mathrm{P}$ $<0.0001)$. Mutational inactivation of the TATA box (mTATA), the Sp1 site (mSp1), or both sites (mSp1mTATA) results in loss of transcriptional activity. B Alternate transcription mechanisms may exist in transcriptional initiation of human AGT. The presence of the Sp1 site $(-70$ to +44$)$ results in promoter activation compared with a promoter with only the TATA box $(-32$ to $+44, \mathrm{P}<0.02)$, or a promoterless control $(\mathrm{P}<0.01)$. Mutational inactivation of the TATA box (mTATA, $\mathrm{P}<0.01)$, the $\mathrm{Sp} 1$ site $(\mathrm{mSp} 1, \mathrm{P}<0.001)$, or both sites (mSp1mTATA, $\mathrm{P}<0.05)$ does not result in complete loss of transcriptional activity
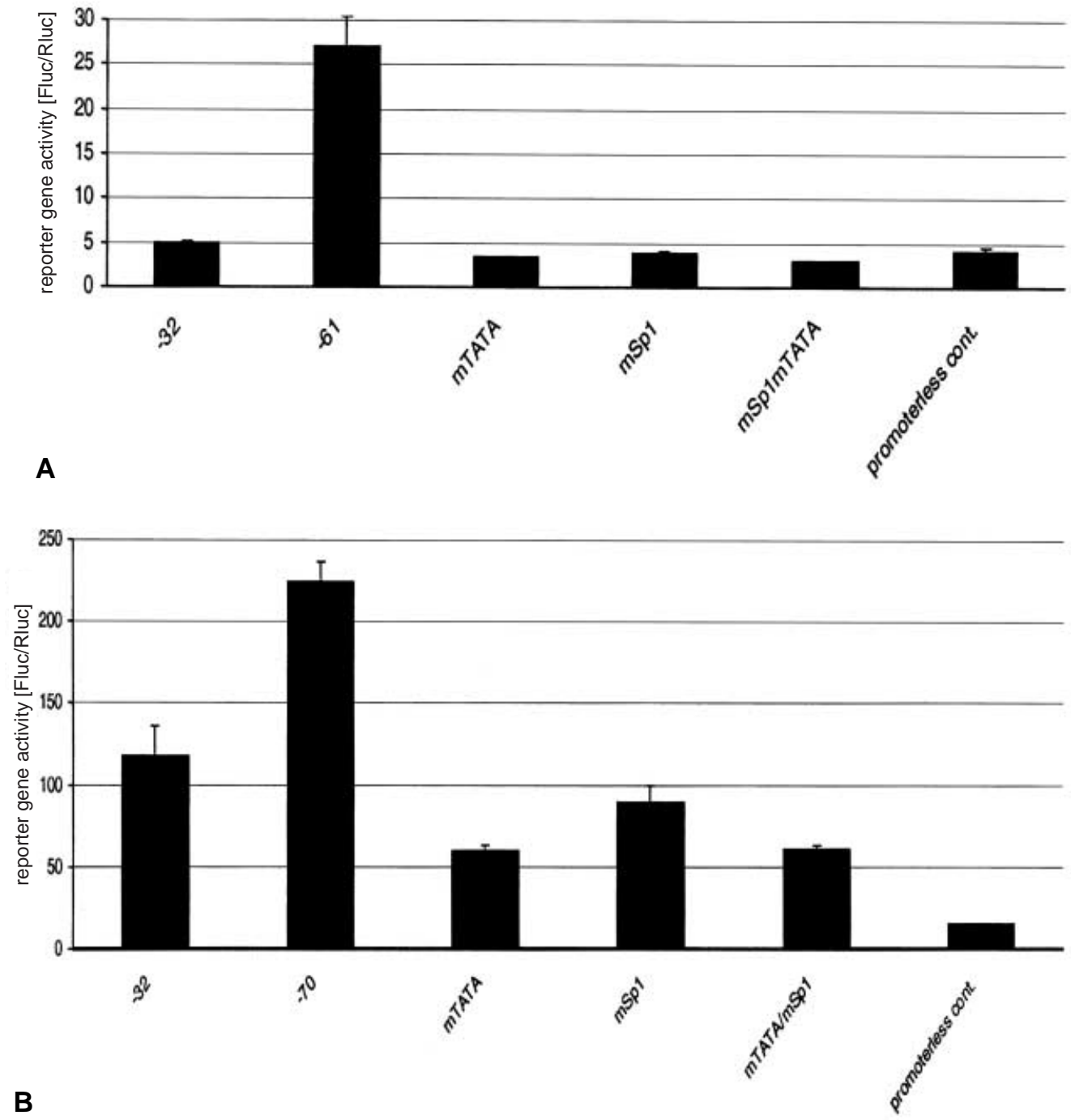

extending $5^{\prime}$ of the TATA box by 31 bp $(-61$ to +44$)$. Similar results were obtained for the mouse proximal tubule cell line (tsMPT) and the hepatoma cell line (Hepa1-6) (Fig. 1). Sequence analysis revealed several transcription factor consensus motifs within this region of transcriptional activation: GATA ( -56 to -51$)$, mycCF1 ( -53 to -48$)$, two NF1 half sites $(-50$ to -47 and -44 to -41$)$, and an Sp1 site $(-47$ to -38$) 5$ bp adjacent to the TATA box. Using in vitro binding experiments, we identified $\mathrm{Sp} 1$ as the factor binding to this region (Figs. 3 and 4).

The fact that a minimal murine AGT promoter ( -32 to $+44)$ is inactive and that a minipromoter spanning the region from -61 to +44 is sufficient for promoter activity led us to entertain the possibility that the Sp1 consensus binding site contributes to transcription initiation of mouse AGT (Fig. 5A). We demonstrated that, within the context of a short AGT promoter, the $\mathrm{Sp} 1$ site $(-47$ to -38$)$ is necessary but not sufficient for murine AGT transcription in vitro. Furthermore, mutational inactivation of the TATA box alone or in combination with the Sp1 binding site indi- cated that the Sp1 consensus motif and the TATA box are necessary but by themselves not sufficient for transcription of the murine $A G T$ gene.

In contrast to the murine AGT promoter, a minimal human AGT promoter $(-32$ to +44$)$ is active, confirming previous observations (Fig. 5B) (Fukamizu et al. 1990). Similar to the mouse promoter, a human AGT promoter construct, including the Sp1 site $(-70$ to +44$)$, is more active when compared with a minimal promoter $(-32$ to $+44)$. Mutational inactivation of the Sp1 consensus motif, the TATA box, or combined inactivation of both sites resulted in reduced transcriptional activity but not in complete transcriptional inactivation of the AGT promoter in vitro. This observation suggests either cell line specific differences or the possibility of alternative, TATA boxindependent, mechanisms of initiation of transcription. The lack of transcriptional inactivation observed for the double mutant suggests yet another TATA box or Sp1-independent mechanism to initiate transcription of human AGT. A potential candidate for this function is the promoter region 
between nucleotides -25 and +1 (Yanai et al. 1997), a region without sequence homology in the mouse promoter. Functional studies by Yanai et al. (1997) have demonstrated that the binding activity has biological and immunological similarity to USF1, a helix-loop-helix nuclear factor (Sawadogo and Roeder 1985), and mutational inactivation of this region leads to decreased promoter activity.

In promoters containing TATA elements, Sp1 interacts with the transcription initiation complex TFIID through specific binding to TATA-associated factors (Chen et al. 1994; Chiang and Roeder 1995; Ryu et al. 1999). Sp1 recruits and stabilizes basal factors through direct interaction with $\mathrm{TAF}_{55}$ and $\mathrm{TAF}_{110}$ (Chen et al. 1994; Chiang and Roeder 1995). When Sp1 is present before and during formation of the preinitiation complex, it increases the amount of closed preinitiation complex assembled (Narayan and Wilson 2000). When Sp1 is present only after the preinitiation complex is formed, it might stimulate transcription by modulation of protein-protein interactions and stimulation of promoter clearance (Narayan and Wilson 2000). However, the precise molecular mechanisms by which Sp1 affects $A G T$ gene transcription can only be investigated using in vitro reconstitution assays. In summary, this study demonstrates significant species-specific differences in AGT promoter function and emphasizes the difficulties in reconstituting the $-6(\mathrm{~A} / \mathrm{G})$ promoter polymorphism in "genetically meaningful" transgenic mouse models. Because of species-specific differences of the -6 region, simple $-6(\mathrm{~A})$ substitution experiments may offer very limited functional information. This study also documents an instance where neither TATA nor Sp1 sites in the proximal promoter of a gene alone are sufficient to promote transcription.

Acknowledgments This work was supported in parts by NIH Grants HL45325 and HL54471. We thank Drs. Tomoaki Ishigami and Barbu Gociman for careful review of the manuscript.

\section{References}

Brasier AR, Tate JE, Ron D, Habener JF (1989) Multiple cis-acting DNA regulatory elements mediate hepatic angiotensinogen gene expression. Mol Endocrinol 3:1022-1034

Chen JL, Attardi LD, Verrijzer CP, Yokomori K, Tjian R (1994) Assembly of recombinant TFIID reveals differential coactivator requirements for distinct transcriptional activators. Cell 79:93-105

Chiang CM, Roeder RG (1995) Cloning of an intrinsic human TFIID subunit that interacts with multiple transcriptional activators. Science 267:531-536

Congiu M, Clouston WM, Fernley RT, Richards RI (1992) The function of conserved elements in the promoter of the mouse angiotensinogen gene. J Mol Endocrinol 9:19-29

Dignam JD, Lebovitz RM, Roeder RG (1983) Accurate transcription initiation by RNA polymerase II in a soluble extract isolated from mammalian nuclei. Nucleic Acids Res 11:1475-1489

Fukamizu A, Murakami K (1997) Activated and inactivated reninangiotensin system in transgenic animals: from genes to blood pressure. Lab Anim Sci 47:127-131

Fukamizu A, Takahashi S, Seo MS, Tada M, Tanimoto K, Uehara S, Murakami K (1990) Structure and expression of the human angiotensinogen gene. Identification of a unique and highly active promoter. J Biol Chem 265:7576-7582

Hata A, Namikawa C, Sasaki M, Sato K, Nakamura T, Tamura K, Lalouel JM (1994) Angiotensinogen as a risk factor for essential hypertension in Japan. J Clin Invest 93:1285-1287

Heinemeyer T, Wingender E, Reuter I, Hermjakob H, Kel AE, Kel OV, Ignatieva EV, Ananko EA, Podkolodnaya OA, Kolpakov FA, Podkolodny NL, Kolchanov NA (1998) Databases on transcriptional regulation: TRANSFAC, TRRD and COMPEL. Nucleic Acids Res 26:362-367

Heinemeyer T, Chen X, Karas H, Kel AE, Kel OV, Liebich I, Meinhardt T, Reuter I, Schacherer F, Wingender E (1999) Expanding the TRANSFAC database towards an expert system of regulatory molecular mechanisms. Nucleic Acids Res 27:318322

Inoue I, Nakajima T, Williams CS, Quackenbush J, Puryear R, Powers M, Cheng T, Ludwig EH, Sharma AM, Hata A, Jeunemaitre X, Lalouel JM (1997) A nucleotide substitution in the promoter of human angiotensinogen is associated with essential hypertension and affects basal transcription in vitro. J Clin Invest 99:17861797

Jeunemaitre X, Soubrier F, Kotelevtsev YV, Lifton RP, Williams CS, Charru A, Hunt SC, Hopkins PN, Williams RR, Lalouel JM, Corvol P (1992) Molecular basis of human hypertension: role of angiotensinogen. Cell 71:169-180

Jones KA, Kadonaga JT, Luciw PA, Tjian R (1986) Activation of the AIDS retrovirus promoter by the cellular transcription factor, Sp1. Science 232:755-759

Kim HS, Krege JH, Kluckman KD, Hagaman JR, Hodgin JB, Best CF, Jennette JC, Coffman TM, Maeda N, Smithies O (1995) Genetic control of blood pressure and the angiotensinogen locus. Proc Natl Acad Sci USA 92:2735-2739

Kriwacki RW, Schultz SC, Steitz TA, Caradonna JP (1992) Sequencespecific recognition of DNA by zinc-finger peptides derived from the transcription factor Sp1. Proc Natl Acad Sci USA 89:97599763

Lifton RP, Gharavi AG, Geller DS (2001) Molecular mechanisms of human hypertension. Cell 104:545-556

Loghman-Adham M, Rohrwasser A, Helin C, Zhang S, Terreros D, Inoue I, Lalouel JM (1997) A conditionally immortalized cell line from murine proximal tubule. Kidney Int 52:229-239

Maxam AM, Gilbert W (1980) Sequencing end-labeled DNA with base-specific chemical cleavage. Methods Enzymol 65:499-560

Morgan T, Craven C, Nelson L, Lalouel JM, Ward K (1997) Angiotensinogen T235 expression is elevated in decidual spiral arteries. J Clin Invest 100:1406-1415

Narayan S, Wilson SH (2000) Kinetic analysis of Sp1-mediated transcriptional activation of a TATA-containing promoter. Biochemistry 39:818-823

Ryu S, Zhou S, Ladurner AG, Tjian R (1999) The transcriptional cofactor complex CRSP is required for activity of the enhancerbinding protein Sp1. Nature 397:446-450

Sawadogo M, Roeder RG (1985) Interaction of a gene-specific transcription factor with the adenovirus major late promoter upstream of the TATA box region. Cell 43:165-175

Tamura K, Tanimoto K, Ishii M, Murakami K, Fukamizu A (1993) Proximal and core DNA elements are required for efficient angiotensinogen promoter activation during adipogenic differentiation. J Biol Chem 268:15024-15032

Tamura K, Umemura S, Ishii M, Tanimoto K, Murakami K, Fukamizu A (1994) Molecular mechanism of transcriptional activation of angiotensinogen gene by proximal promoter. J Clin Invest 93:13701379

Ward K, Hata A, Jeunemaitre X, Helin C, Nelson L, Namikawa C, Farrington PF, Ogasawara M, Suzumori K, Tomoda S, Berrebi S, Sasaki M, Corvol P, Lifton RP, Lalouel JM (1993) A molecular variant of angiotensinogen associated with preeclampsia. Nat Genet 4:59-61

Yanai K, Saito T, Hirota K, Kobayashi H, Murakami K, Fukamizu A (1997) Molecular variation of the human angiotensinogen core promoter element located between the TATA box and transcription initiation site affects its transcriptional activity. J Biol Chem 272: 30558-30562 